Background: Chronic inflammatory rheumatic diseases are risk factors of bone loss and fractures. Systemic sclerosis (SSc) has been recognized to be another potential inflammatory joint disease that may affect bone tissue.

Objectives: to evaluate bone mineral density (BMD) and risk factors of low BMD in women with SSc.

Methods: 173 women, among them 110 postmenopausal (median age 60[55,63] years) and 63 premenopausal (median age 35[31,44] years). BMD was measured at lumbar spine (LS), femoral neck (FN) and total hip (TH) by dual energy X-ray absorptiometry (DXA, Hologic 4500A). Low BMD was diagnosed if the T-score was $<-1.0$ standard deviation (SD) in postmenopausal women and if the Z-score was <-2.0 SD in premenopausal women. The relationship between BMD and SSc patients' characteristics was evaluated using univariate linear regression analysis. Results: Low BMD was found in $66 \%$ patients: $79 \%$ - in postmenopausal and $18 \%$ - in premenopausal women. Among postmenopausal persons osteoporosis was discovered in $47 \%$ and osteopenia - in $32 \%$ cases. In postmenopausal woman BMD of LS, FN and TH were associated with body mass index (BMI) ( $\beta=0.27$, $p=0.010 ; \beta=0.47, p<0,001$ and $\beta=0.45, p<0,001$, respectively), duration of glucocorticoids (GCs) using ( $\beta=-0.31, p=0.008 ; \beta=-0.34, p=0.003$ and $\beta=-0.27, p=0.022$, respectively); BMD of $F N$ and $\mathrm{TH}$ with $\mathrm{C}$-reactive protein $(\beta=-0.32, \mathrm{p}=0.016$ and $\beta=-0.29, p=0.029$, respectively) and LS BMD with current and cumulative GCs dose $(\beta=-0.24, p=0.039$ and $\beta=-0.29, p=0.014$, respectively). In premenopausal women BMD of LS, FN and TH were associated with BMI $(\beta=0.51, p<0,001$; $\beta=0.45, p=0.003$ and $\beta=0.47, p=0.002$, respectively), duration of GCs using ( $\beta=$ $-0.45, p=0.004 ; \beta=-0.47, p=0.003$ and $\beta=-0.48, p=0.002$, respectively) and $G C s$ cumulative dose $(\beta=-0.48, p=0.002 ; \beta=-0.51, p=0.001$ and $\beta=-0.46, p=0.004$, respectively); BMD of FN and TH with $25(\mathrm{OH}) \mathrm{D}$ level $(\beta=0.52, p=0.008$ and $\beta=0.54$, $p=0.005$, respectively), and LS BMD with SSc duration $(\beta=-0.44, p=0.004)$. Conclusion: Low BMD was diagnosed in $66 \%$ of women with SSc. Low BMI, GCs cumulative dose and duration of GCs using were independent risk factors for low BMD in both premenopausal and postmenopausal persons. Additional factors as SSc duration and low vitamin D level were found out for premenopausal and current GCs dose and C-reactive protein level for postmenopausal women.

Disclosure of Interests: None declared

DOI: 10.1136/annrheumdis-2021-eular.1339

\section{POS0842 \\ LUNG ULTRASOUNDTO ASSESS THE SEVERITY OF INTERSTITIAL LUNG DISEASE IN SYSTEMIC SCLEROSIS}

C. Bruni ${ }^{1}$, L. Mattolini ${ }^{1}$, L. Tofani ${ }^{1}$, L. Gargani ${ }^{2}$, N. Landini ${ }^{3,4}$, G. Lepri ${ }^{1}$ M. Orlandi ${ }^{1}$, S. Guiducci ${ }^{1}$, S. Bellando Randone ${ }^{1}$, M. Matucci-Cerinic ${ }^{1}$.

${ }^{1}$ Careggi University Hospital, Experimental and Clinical Medicine, Division of Rheumatology, Firenze, Italy; ${ }^{2}$ National Research Council (CNR) PISA, Institute of Clinical Physiology, Pisa, Italy; ${ }^{3}$ Cà Foncello General Hospital, Radiology, Treviso, Italy: ${ }^{4}$ University of Florence, Experimental and Clinical Biomedical Sciences, Division of Radiology, Firenze, Italy

Background: Interstitial lung disease (ILD) is one of the most common complications and one of the main causes of morbidity and mortality in Systemic Sclerosis (SSc). High-resolution computed tomography (HRCT) is the gold standard for the diagnosis of ILD and it allows its quantification. Among semi-quantitative methods, Goh et al proposed a semi-quantitative scoring system to visually quantify ILD extent, with categorical cut-off of $20 \%$ to distinguish limited and extensive parenchymal involvement with prognostic implications. More recently, the use of radiomics has allowed the objective quantification of ILD through the use of dedicated software, which calculate different parameters of lung density. Given the exposure to ionizing radiation that the procedure entails, other methods of ILD evaluation are being studied, among which lung ultrasound (LUS) identifies the B-lines as a main feature of ILD. So far, different evidences have proposed the use of LUS for the screening of ILD, even in the early phases of the disease and in subclinical lung involvement.

Objectives: the aim of this study is to test the role of LUS in quantifying the severity of SSc-ILD, evaluated with both semi-quantitative visual radiological and quantitative radiomic scores.

Methods: Adult SSc patients classified according to the ACR/EULAR 2013 criteria patients were assessed with pulmonary function test (PFTs), lung ultrasound and HRCT over 60 days. CT images were analysed qualitatively (by presence/absence of ILD), semi-quantitatively (categorical Goh score $<20 \%$ vs $>20 \%$ of extent and the continuous extent Goh score made from 5 levels' assessment- 0 to $100 \%$ ) and quantitatively [with the densitometric radiomic data obtained through the Horos software - Mean lung attenuation (MLA), Standard Deviation (SD), Kurtosis, Skewness and Lung volume (LV)]. LUS was used to quantify the B-lines detected in each patient by scanning a total of 13 intercostal spaces, on both anterior and posterior chest wall.

Results: Among 59 SSc patients ( $81 \%$ women, mean age $48 \pm 14$ years, $45 \%$ anti-Scl70 positive), 23 (39\%) presented ILD on HRCT, of which 14 limited and 9 extensive. The mean visual semi-quantitative score was $6 \%$, ranging from 0 to $66 \%$. Our data showed a significantly different number of B-Lines in ILD vs non-ILD patients (median 38 vs $9, p<.005$ ), a result which was further confirmed among non-ILD vs ILD> 20\% (median 47 vs $9, p=.001$ ) and ILD $<20 \%$ (median
36 vs $9, p=.001$ ) patients. Conversely, the number of B-lines was not statistically different between patients with ILD $<20 \%$ and $>20 \%$ (median 47 vs $36, p=.78$ ). We observed a significant negative correlation between the number of $\mathrm{B}$-lines and FVC ( $r=-.472, p<.05)$ TLC $(r=-.436, p=.003)$, DLco $(r=-.515, p<.001), D L C O /$ VA $(r=.-306, p=.03)$. Finally, the number of $B$-lines showed a statistically significan correlation with the Goh score on 5 levels $(r=437, p=.001)$, MLA ( $r=.571, p<.001)$, kurtosis $(r=-.285, p=.028)$, skewness $(r=-.370, p=.004)$ and LV $(r=-.277, p=.033)$. All data were confirmed analysing anterior and posterior B-Lines separately. Conclusion: Our study confirms that LUS represents a useful tool for the identification of SSc-ILD. In addition, we showed that LUS may be useful also for the quantification of the severity of SSc-ILD, by correlating with PFT parameters, radiomics parameters and visual radiological evaluation. Together with the PFTs, LUS could be used to increase the accuracy of the screening and, potentially, of the follow-up of SSc-ILD patients.

Disclosure of Interests: Cosimo Bruni: None declared, Lavinia Mattolini: None declared, Lorenzo Tofani: None declared, Luna Gargani Consultant of: GE Healthcare, Philips Healthcare and Caption Health, Nicholas Landini: None declared, Gemma Lepri: None declared, Martina Orlandi: None declared, Serena Guiducci: None declared, Silvia Bellando Randone: None declared, Marco Matucci-Cerinic: None declared

DOI: 10.1136/annrheumdis-2021-eular.1350

\section{POS0843 1 A NEW RISK MODEL IS ABLE TO IDENTIFY SYSTEMIC SCLEROSIS PATIENTS WITH A LOW RISK OF DISEASE PROGRESSION}

N. Van Leeuwen ${ }^{1}$, M. Maurits ${ }^{1}$, S. Liem ${ }^{1}$, J. Ciaffi ${ }^{1}$, N. Ajmone-Marsan ${ }^{2}$, M. Ninaber ${ }^{3}$, C. Allaart ${ }^{1}$, H. Gillet-van Dongen ${ }^{4}$, R. Goekoop ${ }^{5}$, T. Huizinga ${ }^{1}$, R. Knevel ${ }^{1}$, J. De Vries-Bouwstra ${ }^{1} .^{1}$ Leiden University Medical Center, Rheumatology, Leiden, Netherlands; ${ }^{2}$ Leiden University Medical Center, Cardiology, Leiden, Netherlands; ${ }^{3}$ Leiden University Medical Center, Pulmonology, Leiden, Netherlands; ${ }^{4}$ Haaglanden Medisch Centrum, Rheumatology, Den Haag, Netherlands; ${ }^{5}$ HagaZiekenhuis, Rheumatology, Den Haag, Netherlands

Background: Disease course in Systemic Sclerosis (SSc) ranges from mild, to severe with progressive organ involvement within months. Guidelines for follow-up are mainly based on expert consensus, and advocate annual assessment. So far, no data driven guidelines exist that describe tailormade systematic assessments for individual patients in line with individual disease course.

Objectives: To develop a prediction model to guide annual assessment of SSc patients tailored in accordance to disease activity.

Methods: A machine learning approach was used to develop a model that can identify patients without disease progression. SSc patients included in the prospective Leiden SSc cohort and fulfilling the ACR/EULAR 2013 criteria were included. The primary endpoint in the prediction model was disease progression which was defined as progression in $\geq 1$ organ system, and/or start of immunosuppression or death between the two most recent visits. Using elastic-net-regularization, and including 90 independent clinical variables (100\% complete), we trained the mode on $75 \%$ and validated it on $25 \%$ of the patients in order to perform internal validation of the final model. We optimized the model on negative predictive value (NPV) to minimize the likelihood of missing progression. By expert assessment of the test characteristics, including swarm plots of the probability scores, cut-offs were identified for low, intermediate and high risk for disease progression.

Results: Of the 492 SSc patients (range of follow-up: 2-10yrs), disease progres sion during follow-up was observed in $52 \%$ (median time $4.9 \mathrm{yrs}$ ), including myocardial progression in $29 \%$, lung progression in $23 \%$, skin progression in $16 \%$,

\begin{tabular}{|c|c|c|c|}
\hline Baseline characteristics & $\begin{array}{c}\text { Total } \\
n=492\end{array}$ & $\begin{array}{c}\text { Non-Progressors } \\
\quad \mathrm{N}=235\end{array}$ & $\begin{array}{l}\text { Progressors } \\
\qquad \mathrm{N}=257\end{array}$ \\
\hline \multicolumn{4}{|l|}{ Demographics } \\
\hline Female, n (\%) & $389(79)$ & $193(82)$ & $196(76)$ \\
\hline Age, mean (SD) & $55(14)$ & $55(15)$ & $55(13)$ \\
\hline Disease duration nonRP, median (IQR) & $3.2(0.9-10.3)$ & $3.5(0.8-10.5)$ & $3.6(1.1-9.3)$ \\
\hline \multicolumn{4}{|l|}{ Organ involvement } \\
\hline DcSSc, n (\%) & $118(24)$ & $34(15)$ & $84(33)$ \\
\hline DLCO $\%$ of pred, mean (SD) & $66(18)$ & $69(18)$ & $64(17)$ \\
\hline FVC\% of pred, mean (SD) & $98(23)$ & $96(24)$ & $97(21)$ \\
\hline ILD on HRCT, n (\%) & $183(37)$ & $66(28)$ & $117(46)$ \\
\hline $\mathrm{PAH}, \mathrm{n}(\%)$ & $26(5)$ & $10(4)$ & $16(6)$ \\
\hline GAVE, n (\%) & $9(2)$ & $4(2)$ & $5(2)$ \\
\hline Cardiac involvement, $\mathrm{n}(\%)$ & $28(6)$ & $14(6)$ & $14(5)$ \\
\hline Myositis, n (\%) & $8(2)$ & $6(3)$ & $2(1)$ \\
\hline Renal crisis, $n(\%)$ & $14(3)$ & $6(3)$ & $8(3)$ \\
\hline \multicolumn{4}{|l|}{ Autoantibodies } \\
\hline Anti-centromere, $\mathrm{n}(\%)$ & $194(39)$ & $118(50)$ & $76(30)$ \\
\hline Anti-topoisomerase, n (\%) & $116(24)$ & $42(18)$ & $74(29)$ \\
\hline
\end{tabular}

$\mathrm{RP}=$ raynaud phenomenon, $\mathrm{dcSSc}=$ diffuse cutaneous systemic sclerosis, $\mathrm{mRSS}=$ modified rod nan skin score, $\mathrm{DU}=$ digital ulcera, $\mathrm{DLCO}=$ single-breath diffusing capacity for carbon monoxide, $\mathrm{FVC}=$ forced vital capacity, ILD=interstitial lung disease, HRCT= high resolution computed tomography, $\mathrm{PAH}=$ pulmonary arterial hypertension, GAVE= gastric antral vascular extasia. 